\title{
Thermal emission in fatigue described by power laws
}

\author{
G. Curti ${ }^{1}$, F. Curà ${ }^{1, a}$, A.E. Gallinatti ${ }^{1}$, and R. Sesana ${ }^{1}$ \\ ${ }^{1}$ Politecnico di Torino, Dipartimento di Meccanica, C.so Duca degli Abruzzi 24, 10129 Torino, Italy
}

\begin{abstract}
In the present work, a theoretical model proposed by the literature and focused on the relationship between microplasticizations thermal behaviour and fatigue scatter is analysed and applied to fatigue test results of standard and notched steel specimens. The same experimental data are subjected to the TCM (Two Curves Method) thermographic elaboration technique, in order to quickly evaluate fatigue limit values. TCM method has been modified, aiming at interpolating thermal data referred to the region of loads upper than fatigue limit with a non linear regression law having the same mathematical structure of the theoretical model equations (power laws).
\end{abstract}

\section{Introduction}

Fatigue investigation, even if begun in the $\mathrm{XIX}^{\circ}$ century, needs still today of rigorous experimental activity.

Aiming at adding more and more information from the tests, it is convenient to use different devices, if possible non destructing, non contacting and real time, to better study some specific aspects of the material behaviour. As an example, infrared thermography is a rather recent technique, able to measure surface thermal increments of fatigued specimens; thermal data may be used to assess the amount of energy dissipations under heat form, on the basis of appropriate theoretical considerations.

A common approach of the thermography in mechanics of materials is the evaluation of the fatigue limit (an example in [1]). The purpose of this research field is to propose a quicker and less expensive methodology than the Stair Case Method (SCM) ([2]).

In particular, in [1], the so called TCM (Two Curves Method) technique is presented, developing the following basic idea. Thermal data, stored in a graph having the test stress on the abscissa axis and a thermographic parameter on the ordinate one, may be divided in two sets, depending on their correspondent stress value (that will be below or above the fatigue limit); then, each set of data may be interpolated by a least square regression line and, finally, the intersection between the two different lines (on the stress axis) corresponds to the fatigue limit. To overcome the difficulty that the fatigue limit is a priori unknown, making impossible to rightly divide the data set, an iterative procedure has been presented in [1]. To this aim, a Matlab® program has been written, dividing the two thermal datasets starting from a first stress value (called First Trial Stress) and directly calculating the intersection of the correspondent regression lines; the stress value (corresponding to the intersection of the regression lines) is generally different from the first trial stress and may be

\footnotetext{
a e-mail : francesca.cura@polito.it
} 
chosen as the new trial stress value of the second iteration, and so on. This sequence has to be repeated until the convergence occurs.

More recently, an alternative to this procedure was proposed, substituting the second regression line (referred to loading values above the fatigue limit) with an exponential curve [3], [4] (non linear regression). This modification to the original TCM version is justified not only by the improved statistical regression coefficients (determination and robustness ones) but, in the Authors opinion, also because the material thermal behaviour in that region could better be modelled by non linear relationships, how suggested, as an example, by a theoretical law available in literature $[5,6]$.

In that work [5], a probabilistic model (a Poisson Point Process) has been presented to describe the intensity of the microplasticizations activation process, deducing from this law the global energy dissipated under heat form during a fatigue cycle; finally, thanks to the integration of the conduction equation, the relationship between stabilization (or steady state) temperature and fatigue load has been obtained; both quantities depend on fatigue load raised to a power which is a function of a material parameter.

Since the microplasticizations activation is relevant for loads upper than the fatigue limit, it may be supposed that such power model [5] could rightly represent thermal data referred to this stresses range. On the contrary, in the Authors opinion, for loading levels lower than fatigue limit the material behaviour (and the corresponding heat dissipation) is influenced more by anelasticity (considered in general a linear phenomenon) than by microplasticizations activation ([3],[7-9]). As a consequence of that, being the power model less suitable to describe thermal data corresponding to stress values lower than the fatigue limit, the modified TCM method uses a line interpolating the first dataset and a non linear curve for the second one.

The first aim of the present paper is to apply the basic law discussed in [5] (used on smooth specimens) and its coefficients (depending on the material) to different kinds of steels and specimens, comparing the obtained results in terms of fatigue limit values to those coming out from the modified TCM technique, where a power regression curve of the same structure has been implemented. Then, for the same test cases, some new thermal parameters as subtended areas and temperatures at a fixed number of cycles ([3], [4]) are considered in both model and processing procedure, instead of the traditional stabilization (steady state) temperature utilised in [5].

The final aim of the work is to investigate if the theoretical approach presented in [5] can justify the reliability of the fatigue limit estimations obtained by the modified TCM method.

Firstly, the dual phase steel (DP60) analysed in [6] is considered and the corresponding data processed. Then, two steels are taken into account, a C45 steel, standard specimens, and a 25CrMo4 steel, notched specimens with semicircular notch (notch radius $1.4 \mathrm{~mm}$ ). Experimental data refer to uniaxial symmetric alternate sinusoidal fatigue tests performed at the Laboratory of the Dipartimento di Meccanica (Politecnico di Torino).

\section{Reference theoretical model}

In the present paragraph, the probabilistic two scale model discussed in [5,6] and other papers will be rapidly summarised (for sake of brevity).

In this model, it is proposed a law describing the microplasticization activity in a metallic material subjected to $\mathrm{HCF}$ (High Cycles Fatigue), treating the microplasticizations as some inclusions in the elastic matrix of the material following a Poisson Point Process of intensity $\lambda$; in particular, this last parameter may be calculated from the law here reported:

$$
\lambda\left(\Sigma_{e}\right)=\frac{1}{V_{0}}\left(\frac{\Sigma_{0}}{S_{0}}\right)^{m}
$$

that corresponds to a Weibull model and where $V_{0}$ is the inclusion volume, $m$ is the shape parameter and $V_{0} S_{0}{ }^{m}$ the scale parameter ( $m$ and $S_{0}$ depend on the material).

Developing the free energy associated to an elasto-plastic inclusion, it is possible to evaluate the global dissipated energy density in a cycle: 


$$
\Delta\left(\Sigma_{0}\right)=\frac{4 m}{h(m+1)(m+2)} \frac{\Sigma_{0}^{m+2}}{S_{0}^{m}}
$$

that takes place as heat dissipation, responsible for the temperature increase on the specimen surface. Solving the heat conduction equation, i.e.:

$$
\dot{\Theta}+\frac{\Theta}{\tau}=\frac{\Delta\left(\Sigma_{0}\right) f_{r}}{\rho c}
$$

a relationship between the steady state or stabilization temperature $\bar{\Theta}$ and the alternate stress component $\Sigma_{0}$ can be found:

$$
\Delta T s t a b=\bar{\Theta}=\frac{4 f_{r} \tau m}{h \rho c(m+1)(m+2)} \frac{\sum_{0}^{m+2}}{S_{0}^{m}}
$$

where $f_{r}$ is the load frequency, $\tau$ is the characteristic time, $h$ is the thermal convection coefficient, $\rho$ is the density coefficient and $c$ is the specific heat. The $m$ coefficient may be identified by interpolating some experimental thermal data. To this aim, in [6], it is proposed to perform a so called self heating test on a specimen, consisting in applying repeated blocks of cycles with increasing loading values. As referred in [5], the intensity $\lambda$ characterises both thermal behaviour and fatigue scatter (as a consequence of the weakest link theory [5]), so this last quantity can be assessed from the $m$ coefficient and other ones (dependent on the material); in particular, the probability $P_{F}$ of finding at least one active inclusion in the volume $V_{\Omega}$ can be expressed as:

$$
P_{F}=1-\exp \left(-\lambda V_{\Omega}\right)=1-\exp \left[-\frac{V_{\Omega}}{V_{0}}\left(\frac{\Sigma_{0}}{S_{0}}\right)^{m}\right]=1-\exp \left[-\frac{V_{\Omega} H_{m}}{V_{0}}\left(\frac{\Sigma_{F}}{S_{0}}\right)^{m}\right]
$$

where $H_{m}$ is the stress heterogeneity factor, $V_{\Omega}$ is the whole volume of the calibrated section and $\Sigma_{F}$ is the maximum stress in $V_{\Omega}$.

On the basis of the above quoted theory, the mean fatigue limit may be written as:

$$
\bar{\Sigma}_{\infty}=\frac{S_{0} V_{0}^{1 / m}}{V_{e f f}} \Gamma\left(1+\frac{1}{m}\right)
$$

while the corresponding scatter is:

$$
\bar{\Sigma}_{\infty}^{2}=S_{0}^{2}\left(\frac{V_{0}}{V_{e f f}}\right)^{2 / m}\left[\Gamma\left(1+\frac{2}{m}\right)-\Gamma^{2}\left(1+\frac{1}{m}\right)\right]
$$

where $\Gamma$ is the Gamma Euler's Function and the effective volume $V_{\text {eff }}$ corresponds to:

$$
V_{\text {eff }}=V_{\Omega} H_{m}
$$

The effective volume of a specimen is the whole volume $V_{\Omega}$ of the calibrated section corrected by means of the stress heterogeneity factor $H_{m}$ due to the effective stress distribution.

Being the coefficient $m$ known, the fatigue scatter may be finally evaluated; then, by using the Stromeyer's Law (where $A_{d}$ is a material dependent parameter), both number of cycles to failure at the considered stress level $\Sigma_{0}$ and probability value may be calculated:

$$
N=\frac{A_{d}}{\bar{\Sigma}_{\infty}\left(\Sigma_{0}-\bar{\Sigma}_{\infty}\right)}
$$

In order to resume the theoretical procedure above described, it may be conclude that in general, if experimental data are available referring to a Wöhler curve and a self heating test (only one specimen), it is possible to determine both fatigue limit (associated to the $50 \%$ of probability) and $A_{d}$ and $m$ parameters.

In conclusion, the previous relationships may be used, at this point, also to predict the fatigue limit values and the Wöhler Curves associated to other different failure probabilities.

More details about constants and identification procedure will be done in Paragraph 4. 


\section{Materials}

The basic law in Equation 4 involving the microplasticizations activation process has been utilised to analyse three groups of experimental data, as resumed in Table 1.

In particular, the first data group (second column, Table 1) refers to a dual phase DP60 steel whose experimental analysis is available in [6]. The other two data groups (third and fourth column, Table 1) refer to a research activity carried on at the Dipartimento di Meccanica, Politecnico di Torino, involving respectively $\mathrm{C} 45$ (standard specimens) and $25 \mathrm{CrMo} 4$ (notched specimens) steels (testing machine: Vibroforo Amsler 10 HFP 422; thermotracer: NEC TH7102WX) [3]. These last experimental data refer to fatigue tests performed simultaneously to the acquisitions of surface thermal profiles and may be represented both as Wöhler curves or as graphs showing the stabilization temperature of each specimen (ordinates) plotted versus the corresponding alternate stress level (abscissas); specimen where stressed until failure or $2 * 10^{6}$ cycles [3].

With respect to the three materials above quoted, Table 1 reports: ultimate strength, yield stress, notch radius, test frequency and specimens number (which is different, depending on the thermographic parameter, see Table 1). Standard and notched specimens have been tested.

Table 1. Tested materials.

\begin{tabular}{|c|c|c|c|}
\hline Material & DP60 Steel & C45 Steel & 25CrMo4 Steel \\
\hline Experimental Activity & ENS, CACHAN [6] & Politecnico di Torino & Politecnico di Torino \\
\hline Ultimate strength [MPa] & $>600$ & 800 & 461 \\
\hline Yield stress [MPa] & $>350$ & 500 & 407 \\
\hline Notch radius [mm] & Fillet, $100 \mathrm{~mm}$ & $\infty$ & 1.4 \\
\hline Test frequency [Hz] & 10 & 100 & 100 \\
\hline Specimens number [-] & $\begin{array}{l}15 \text { (Wöhler Curve) } \\
1 \text { (Thermal Data) }\end{array}$ & $\begin{array}{l}15 \text { (Wöhler Curve) } \\
\text { Thermal Data: } 17 \text { ( } \triangle T \text { stab) } \\
49 \text { ( } \triangle T, \text { Area } 50000 \text { cycles) } \\
51 \text { ( } \Delta T, \text { Area } 5000 \text { cycles })\end{array}$ & $\begin{array}{l}24 \text { (Wöhler Curve) } \\
16 \text { (Thermal Data) }\end{array}$ \\
\hline
\end{tabular}

\section{Data processing procedure}

Three groups of experimental data (previously quoted) have been processed following both procedures described in $[5,6]$ and utilised in $[1,3,4]$.

Firstly, a Matlab ${ }^{\circledR}$ program has been developed to implement the theoretical model $[5,6]$, to calculate the related coefficients and to obtain the fatigue limit value (together with its scatter). Then, thermal data coming from the self-heating test (see Paragraph 3) have been processed by means of a non linear regression, following a power law as:

$$
y=a x^{b}
$$

The non linear regression has been performed by utilising the Matlab® toolbox Curve Fitting (Trust Region non linear algorithm). The calculated coefficients are the multiplying constant $a$ and the exponent $b$, useful to easily obtain the shape parameter $m(m=b-2)$.

Then, the Wöhler curve data have been treated by a linear regression operation (ascissa: $1 / \mathrm{N}$, where $N$ is the cycles number; ordinate: alternate stress $\Sigma_{0}$ ). In particular, the fatigue limit and a parameters $A_{d}$ (depending on the material) of the Stromeyer's Law have been calculated (Equation (9)). So, the effective volume of a specimen has to be determined referring to the procedure reported in Appendix A [6], adaptable to specimens with different geometries. Then, it may be possible to obtain the scale parameter $S_{0} V_{0}{ }^{1 / m}$ by utilising Equation (6). The constant $\eta V_{0}$ describing the thermal kinetic (see Equation (4)) due to fatigue stresses may be expressed as a function of the regression coefficient $a$ :

$$
\eta V_{0}=\frac{4 f_{r} \tau}{h \rho C_{\varepsilon, \alpha}} V_{0}=a \frac{(m+1)(m+2) V_{0} S_{0}^{m}}{m}
$$


Thermal data have also treated by means of the TCM procedure, in order to estimate the fatigue limit value. TCM procedure, described in detail in $[3,4]$, has been adapted to the aim of the present paper, that is to process the same data groups by means of two different methodologies.

It may be noted that not only the stabilization temperature has been utilised as thermal parameter, but also subtended areas and temperatures at a fixed number of cycles (below and above the stabilization temperature) have been considered.

In order to verify the obtained results, fatigue limit values have been determined (where possible) by means of the Stair Case Method [2].

\section{Results and Remarks}

The results of the present paper are resumed in Tables 2, 3, 4 and 5 .

In order to check Matlab® M-File written to automatically calculate the coefficients of the reference theoretical model [5,6], firstly the dual phase ferrite-martensite steel DP60 has been analysed, whose data have been extrapolated from the diagrams in $[5,6]$.

Table 2 reports for each material (DP60, C45 and 25CrMo4) the fatigue limit value $\sigma_{D-1} 50 \%$ obtained by means of the SCM (corresponding to the $50 \%$ of failure probability and the related scatter) and by means of the reference theoretical model (named Doudard Method, DM) (whose coefficients have been calculated by using the Matlab® M-File). Table 2 reports also the model parameters (the shape parameter $m$, the coefficient $\eta V_{0}$ describing the kinetic of the thermal phenomena, the scale parameter $V_{0} S_{0}{ }^{m}$, and so on) and the intermediate data useful to complete the calculations (as an example, the effective volume $V_{\text {eff }}$ ).

It may be observed that for the steel DP60 the Stair Case results are not available, but in this case in Table 2 are showed the outcomes as reported in [6] (column DM [6]).

The results reported in Tables 3-5 involve the TCM procedure implementation, on the basis of the methodology described in the previous paragraph: the mean fatigue limit has been assessed using the power law fitting and the linear least square regression on two subsets of the thermal data already utilised (see Table 2). When possible (i.e., excepting DP60 steel, due to lack of data), this operation has been repeated for three thermographic parameters as steady state or stabilization temperature $\Delta T s t a b$, subtended area $A$ and temperature increase at a fixed number of cycles $\Delta T$, considering two different number of cycles as reference (the first before and the second after stabilization region).

In particular, Tables 3, 4, 5 (involving respectively Dual Phase DP60, C45 and 25CrMo4 steels) present both estimated fatigue limit, $\sigma_{D-1} 50 \%$, and regression coefficients of the power curve (in the column called TCM). The scatter results, calculated following [3], are not here presented since they are not the main object of this work. In the same tables, for sake of convenience, the already calculated regression coefficients following [5,6] and shown in Table 2 have been reported in the column DM.

The comparison between the two regression procedures is also graphically emphasised, thanks to some graphs (one for each material, considering the thermographic parameter $\Delta T$ stab; for C45 steel, also the subtended area to 50000 cycles has been proposed) showing the TCM regression curves and the power law from [5,6], together with the experimental data (Figures from 1 to 4 ).

On the basis of the previous results, it may be firstly observed that the reference theoretical method has been satisfactory used and reasonable coefficients have been obtained (see Table 2).

In particular, fatigue limit value (treating the procedures the same kind of data) is almost the same using both Stair Case method (SCM), which is preferred at the Politecnico di Torino, and Wöhler Curve elaboration [5,6], only excepting $25 \mathrm{CrMo} 4$ steel, for which it was impossible to rightly calculate the $S C M$ scatter [2].

Secondly, the obtained fatigue limit values from TCM technique (see Tables from 3 to 5) may be judged as acceptable, examining both classical thermographic parameters (i.e. the steady state or stabilization temperature $\Delta T s t a b$ ) and new ones (subtented area and temperature at a fixed number of cycles). In particular, the assessed values are very close to the Stair Case results (here considered the true ones), in the $25 \mathrm{CrMo} 4$ steel case for all the thermographic parameters, and in $\mathrm{C} 45$ steel case for 
the three parameters $\Delta T$ stab, $A$ and $\Delta T$ at 5000 cycles, whereas the ones evaluated at 50000 cycles fairly underestimated them. Small discrepancies in DP60 steel may be due to the experimental data rough assessment coming out from the graphs in [6].

Table 2. Reference model coefficients for the tested materials.

\begin{tabular}{|l|l|l|l|l|l|l|l|}
\hline & \multicolumn{3}{|c|}{ DP60 } & \multicolumn{3}{c|}{ C45 } & \multicolumn{2}{c|}{ 25CrMo4 } \\
\hline Coefficient & $S C M$ & $D M$ & $D M[6]$ & $S C M$ & $D M$ & $S C M$ & $D M$ \\
\hline$\sigma_{D-150 \%}[\mathrm{MPa}]$ & - & 278 & 275 & 239 & 237 & 108 & 116 \\
\hline Scatter $[\mathrm{MPa}]$ & - & 16.22 & Not specified & 9.00 & 25.00 & - & 49.78 \\
\hline$m[-]$ & - & 21.30 & 20 & - & 11.48 & - & 2.54 \\
\hline$b[-]$ & - & 23.30 & 22 & - & 13.48 & - & 4.54 \\
\hline$a[-]$ & - & $5.32 \mathrm{e}-058$ & Not specified & - & $1.87 \mathrm{e}-031$ & - & $1.67 \mathrm{e}-09$ \\
\hline$A_{d}\left[\mathrm{MPa}^{2}\right]$ & - & $8.97 \mathrm{e}+08$ & $1.2 \mathrm{e}+09$ & - & $2.85 \mathrm{e}+08$ & - & $1.58 \mathrm{e}+08$ \\
\hline$\sigma_{S}\left[\mathrm{MPa}^{2}\right]$ & - & 29943 & Not specified & - & 16892 & - & 12576 \\
\hline$V_{e f f}\left[\mathrm{~mm}^{3}\right]$ & - & 593 & 620 & - & 3210 & - & 120 \\
\hline$V_{0} S_{0}^{m}\left[\mathrm{MPa}^{m} \mathrm{~mm}^{3}\right]$ & - & $1.18 \mathrm{e}+055$ & Not specified & - & $1.10 \mathrm{e}+031$ & - & $2.35 \mathrm{e}+07$ \\
\hline$S_{0} V_{0}^{1 / m}\left[\mathrm{MPamm}^{3 / m}\right]$ & - & 385 & 390 & - & 500 & - & 889 \\
\hline$\eta V_{0}\left[{ }^{\circ} \mathrm{Kmm}^{3} \mathrm{MPa}^{-2}\right]$ & - & $1.5 \mathrm{e}-01$ & $1.1 \mathrm{e}-01$ & - & 30 & - & 0.25 \\
\hline
\end{tabular}

Table 3. Dual Phase DP60 steel results.

\begin{tabular}{|l|l|l|}
\hline \multicolumn{2}{|c|}{ Dual Phase Steel DP60 $-\mathbf{1 0 ~} \mathbf{~ H z}-\mathbf{r}=\mathbf{1 0 0} \mathbf{~ m m}$} \\
\hline Coefficient & \multicolumn{2}{|c|}{$\mathbf{C}]$} \\
\hline$\sigma_{D-150 \%}[\mathrm{MPa}]$ & - & 254 \\
\hline$m[-]$ & 21.30 & - \\
\hline$b[-]$ & 23.30 & 24.03 \\
\hline$a[-]$ & $5.32^{\mathrm{e}}-058$ & $1.05 \mathrm{e}-059$ \\
\hline
\end{tabular}

Table 4. C45 steel results.

\begin{tabular}{|c|c|c|c|c|c|c|c|c|c|c|}
\hline \multicolumn{11}{|c|}{$\mathrm{C} 45-100 \mathrm{~Hz}-\mathrm{r}=\infty$} \\
\hline & \multicolumn{2}{|c|}{$\Delta$ Tstab $\left[{ }^{\circ} \mathrm{C}\right]$} & \multicolumn{2}{|c|}{$\begin{array}{l}\text { Area 50000 } \\
{\left[{ }^{\circ} \mathbf{C}^{*} \text { cycles] }\right]}\end{array}$} & \multicolumn{2}{|c|}{$\Delta \mathrm{T50000}\left[{ }^{\circ} \mathrm{C}\right]$} & \multicolumn{2}{|c|}{$\begin{array}{c}\text { Area 5000 } \\
{\left[{ }^{\circ} \mathbf{C}^{*} \text { cycles] }\right.}\end{array}$} & \multicolumn{2}{|c|}{$\Delta \mathrm{T5000}\left[{ }^{\circ} \mathrm{C}\right]$} \\
\hline Coefficient & $D M$ & $T C M$ & $D M$ & $T C M$ & $D M$ & $T C M$ & $D M$ & $T C M$ & $D M$ & $T C M$ \\
\hline$\sigma_{D-150 \%}[M P a]$ & & 234 & & 215 & & 213 & & 238 & & 241 \\
\hline$m[-]$ & 11.48 & - & 3.70 & - & 4.65 & - & 1.30 & - & 2.01 & - \\
\hline$b[-]$ & 13.48 & 18.03 & 5.70 & 5.87 & 6.65 & 6.76 & 3.30 & 4.74 & 4.01 & 5.61 \\
\hline$a[-]$ & $\begin{array}{l}1.87 \mathrm{e}- \\
031\end{array}$ & $\begin{array}{l}2.47 \mathrm{e}- \\
042\end{array}$ & $\begin{array}{l}2.33 \mathrm{e}- \\
08\end{array}$ & $\begin{array}{l}9.17 \mathrm{e}- \\
09\end{array}$ & $\begin{array}{l}3.67 \mathrm{e}- \\
015\end{array}$ & $\begin{array}{l}1.98 \mathrm{e}- \\
015\end{array}$ & $\begin{array}{l}1.90 \mathrm{e}- \\
04\end{array}$ & $\begin{array}{l}6.75 \mathrm{e}- \\
08\end{array}$ & $\begin{array}{l}1.36 \mathrm{e}- \\
09\end{array}$ & $\begin{array}{l}1.93 \mathrm{e}- \\
13\end{array}$ \\
\hline
\end{tabular}

Thirdly, even if the non linear regression has been executed on datasets not equals between them, the regression coefficients of the two power laws assessed following [5,6] or TCM method (shown in the Tables from 3 to 5) are quite similar. This is also evident from the graphs reported in the present paper, where the two plotted power laws are very closed each other. It may be further noted 
that data referred to loads below the fatigue limit are better interpolated using a line (see the dash line) than the power law of [5,6] (represented by the dash dot curve), above all if the materials are stressed using a high frequency load (100 Hz, C45 and 25CrMo4 steels). The power law outcome is, on the contrary, satisfactory for the data in the region of stresses above the fatigue limit.

Table 5. 25CrMo4 steel results.

\begin{tabular}{|c|c|c|c|c|c|c|c|c|c|c|}
\hline \multicolumn{11}{|c|}{$25 \mathrm{CrMo4}-100 \mathrm{~Hz}-\mathrm{r}=1.4 \mathrm{~mm}$} \\
\hline \multirow[b]{2}{*}{ Coefficient } & \multicolumn{2}{|c|}{$\Delta$ Tstab $\left[{ }^{\circ} \mathrm{C}\right]$} & \multicolumn{2}{|c|}{$\begin{array}{l}\text { Area } 60000 \\
{\left[{ }^{\circ} C^{*} \text { cycles }\right]}\end{array}$} & \multicolumn{2}{|c|}{$\Delta \mathrm{T60000}\left[{ }^{\circ} \mathrm{C}\right]$} & \multicolumn{2}{|c|}{$\begin{array}{c}\text { Area } 9050 \\
{\left[{ }^{\circ} \mathbf{C}^{*} \text { cycles }\right]}\end{array}$} & \multicolumn{2}{|c|}{$\Delta \mathrm{T} 9050\left[{ }^{\circ} \mathrm{C}\right]$} \\
\hline & $D M$ & $T C M$ & $D M$ & $T C M$ & $D M$ & $T C M$ & $D M$ & $T C M$ & $D M$ & $T C M$ \\
\hline$\sigma_{D-150 \%}[M P a]$ & - & 105 & - & 106 & - & 106 & - & 105 & - & 106 \\
\hline$m[-]$ & 2.54 & - & 2.48 & - & 2.55 & - & 1.83 & - & 2.18 & - \\
\hline$b[-]$ & 4.54 & 4.74 & 4.48 & 4.73 & 4.55 & 4.78 & 3.83 & 4.01 & 4.18 & 4.40 \\
\hline$a[-]$ & $\begin{array}{l}1.67 \mathrm{e} \\
-09\end{array}$ & $\begin{array}{l}\text { 6.31e- } \\
010\end{array}$ & $\begin{array}{l}1.15 \mathrm{e}- \\
04\end{array}$ & $\begin{array}{l}3.26 \mathrm{e}- \\
05\end{array}$ & $\begin{array}{l}1.61 \mathrm{e} \\
-09\end{array}$ & $\begin{array}{l}5.15 \mathrm{e}- \\
010\end{array}$ & $\begin{array}{l}2.01 \mathrm{e} \\
-04\end{array}$ & $\begin{array}{l}8.54 \mathrm{e}- \\
05\end{array}$ & $\begin{array}{l}6.03 \mathrm{e}- \\
09\end{array}$ & $\begin{array}{l}2.07 \mathrm{e} \\
-09\end{array}$ \\
\hline
\end{tabular}

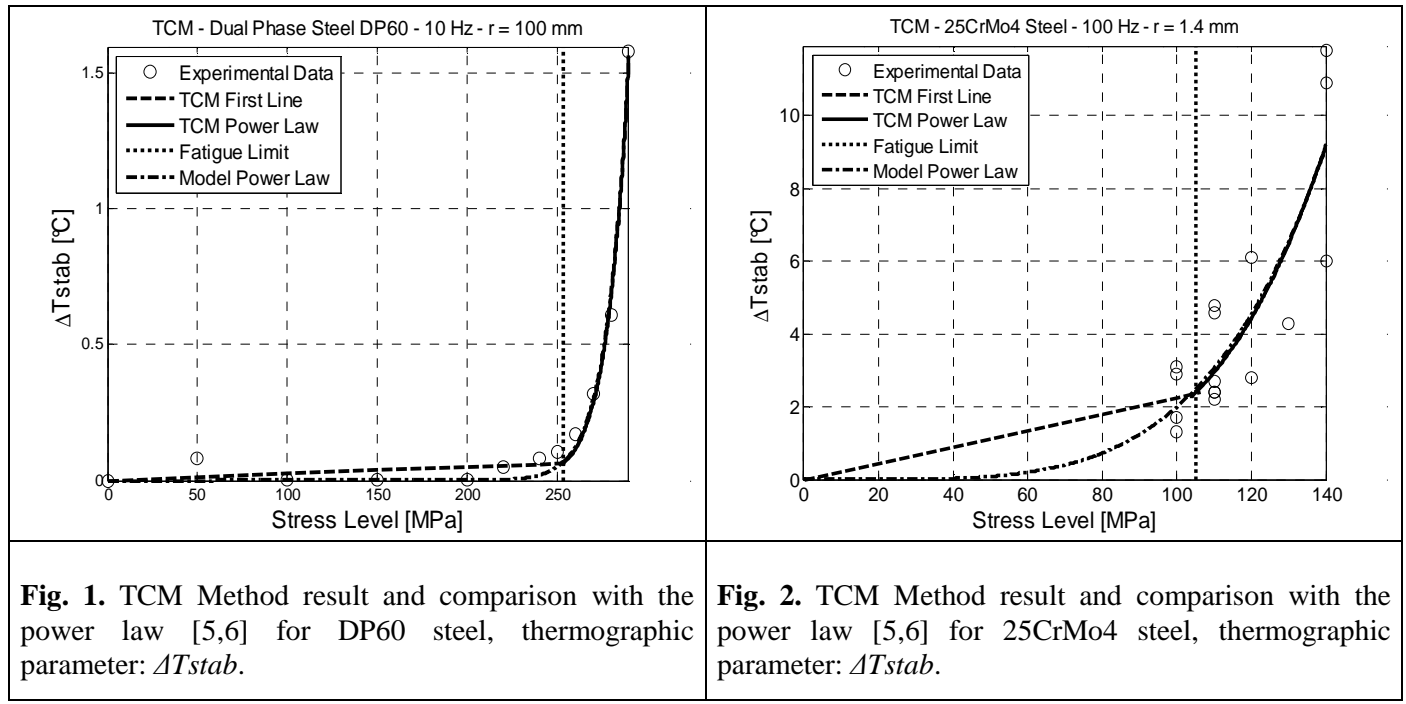

\section{Conclusions}

The obtained results allow us to draw the following conclusions.

The reference power law, previously analysed and then applied in the present work to experimental data referred to three different steels, has provided satisfactory results above all for as concerns stress values above the fatigue limit.

In particular, both fatigue limit values and coefficient related to the theoretical model have been calculated with good approximation.

This reference law has been already applied in literature considering the stabilization temperature as traditional thermal variable. The same procedure, related to the power law, has been adapted in the present work to other thermal parameters, as subtended areas and temperatures at a fixed number of cycles, before and after the above quoted stabilization temperature.

Results coming out from TCM techniques are very good if experimental data corresponding to loading values remarkable higher than the fatigue limit have been interpolated by means of a non linear function (exponential), since the two lines interpolation may overestimate the final result. 
In particular, it may be observed that the data interpolation above the fatigue limit has been performed using a power function having the same structure of the theoretical law available in literature and the corresponding outcomes were acceptable, both concerning the fatigue limit and regression coefficients.

So, the TCM procedure implemented in this work could be physically justified by considering the fatigue limit value as the gradual passage between two regions. The first region (stresses below the fatigue limit) involves linear anelastic phenomena (like the well known boundary grain friction) that are the most relevant, while microplasticizations are negligible; in this case it is acceptable the use of a line to represent the corresponding thermal data. In the second region (stresses above the fatigue limit), on the contrary, microplasticization activation is more and more predominant and the use of a power law is the most suitable. Relevant microplasticizations activation may be considered as one of the mechanisms involving crack initiation, i.e. the first step toward fatigue failure.

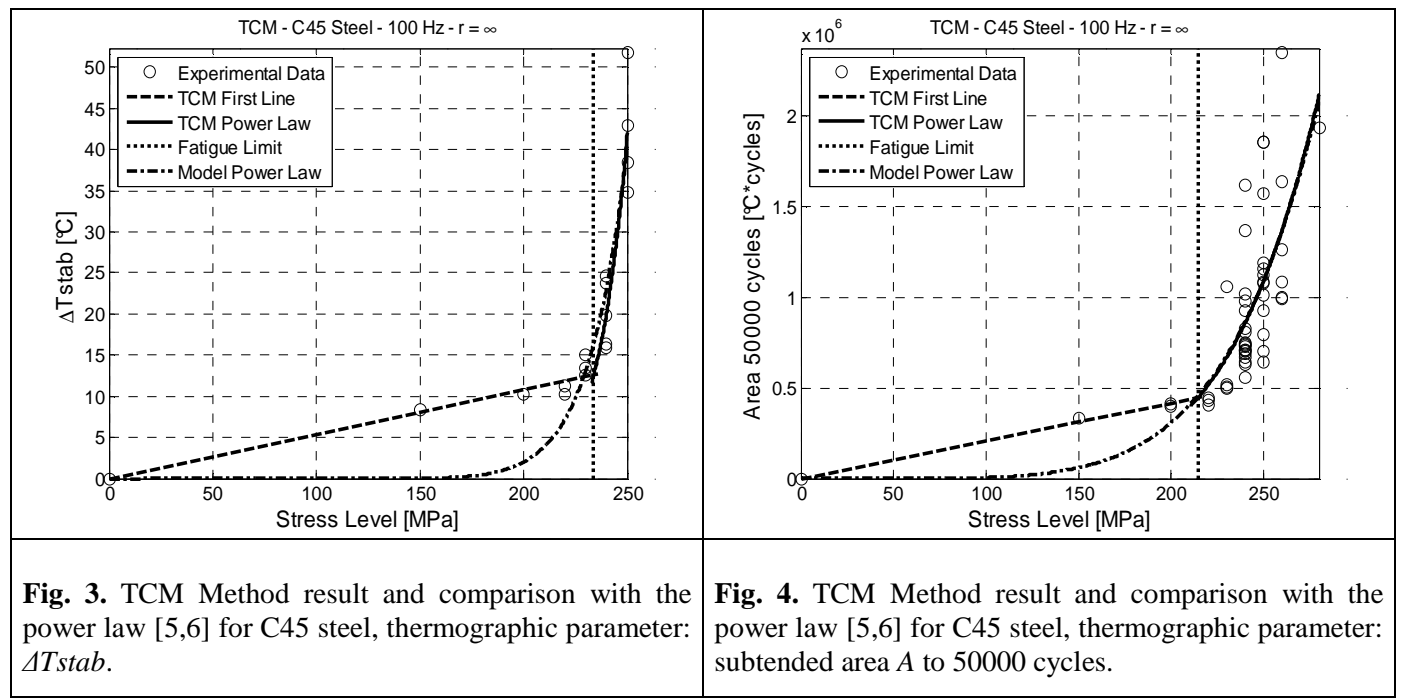

\section{References}

1. F. Curà, G. Curti, R. Sesana, A new iteration method for the thermographic determination of fatigue limit in steels, International Journal of Fatigue, 27, 4, 453-459 (2005)

2. UNI 3964-1984 Standard, Prove meccaniche dei materiali metallici - Prove di fatica a temperatura ambiente - Principi generali, in Italian (1984)

3. A.E. Gallinatti, Theoretical and experimental study of the thermal emission in metallic materials subjected to fatigue loads, PhD Thesis, Politecnico di Torino, Torino (Italy) (2009)

4. F. Curà, G. Curti, A.E. Gallinatti, Limite di fatica con metodi termografici mediante regressione esponenziale, XXXVIII Convegno AIAS, Torino, in Italian $\left(9^{\mathrm{TH}}-11^{\mathrm{TH}}\right.$ September 2009)

5. C. Doudard, S. Calloch, F. Hild, P. Cugy, A. Galtier, Identification of the scatter in high cycle fatigue from temperature measurement, Comptes Rendus de Mécanique 332, 795 - 801 (2004)

6. C. Doudard, S. Calloch, F. Hild, P. Cugy, A. Galtier, A probabilistic two scale model for high cycle fatigue life predictions, Fatigue and Fracture of engineering materials and structures, 28, 3, 279-288 (2005)

7. M.P. Luong, Infrared thermographic scanning of fatigue in metals, Nuclear engineering and design, 158, 363-376 (1995)

8. B.J. Lazan, Damping of materials and members in structural mechanics, Pergamon Press (1968)

9. C. Zener, Élasticité et anelasticité des métaux, Dunod, Paris, in French (1952) 